\title{
VARIATIONS DE LA CONSOMMATION SPONTANÉE D'ÉNERGIE EN FONCTION DE LA NATURE ET DE LA QUANTITÉ DES MATIERES AZOTÉES INGÉRÉES CHEZ LE RAT EN CROISSANCE
}

\author{
A. RERAT et Y. HENRY \\ Station de Recherches sur l'Elevage des Porcs, \\ Centre national de Recherches zootechniques, Fouy-en-fosas (Seine-et-Oise).
}

Il est bien connu que le niveau de consommation des animaux en croissance présente d'importantes variations liées à la nature du régime. Cependant, la question reste ouverte de savoir quel en est le déterminisme. Différentes hypothèses peuvent être émises : on peut supposer que la consommation ne suit aucune règle précise ou, au contraire, qu'elle subit une régulation telle qu'est assurée la couverture de certains des besoins de l'animal, besoin énergétique ou besoin azoté par exemple. Divers travaux semblent prouver le bien fondé de l'hypothèse selon laquelle le niveau de consommation serait réglé par l'animal sur la couverture de son besoin énergétique. Il a été montré par ailleurs que la consommation de nourriture est également fonction de la qualité et du taux de protéines des régimes et on pourrait penser que le niveau de consommation est ajusté par l'animal sur la couverture de son besoin azoté. Ces résultats apparemment contradictoires ne peuvent être compris que si l'on admet que le besoin énergétique varie en fonction de la vitesse de croissance, c'est-à-dire du niveau de la dépense azotée, ellemême réglée par l'apport azoté.

La validité de cette hypothèse ne peut pas être démontrée à l'aide de régimes mixtes où les deux facteurs, azote et énergie, sont associés. Pour y parvenir, il est nécessaire de distribuer séparément, mais simultanément, aliments énergétiques et aliments azotés. Utilisant cette technique, CALET et al. (1961) ont pu montrer que la consommation spontanée d'énergie, à apport azoté égal, est plus élevée pour les protéines de haute valeur biologique que pour les protéines de plus faible valeur biologique. Sur le principe, la méthode utilisée par ces auteurs est très proche d'une méthode pratique d'alimentation utilisée chez le porc : le système Lehmann, basé sur l'administration d'une quantité fixe (imposée) d'un aliment concentré riche en azote et d'une quantité variable (ad libitum) d'un aliment énergétique à faible taux azoté (orge par exemple). L'amélioration de ce système pourrait permettre de préciser chez le porc, l'équilibre optimum entre les fractions azotée et énergétique des régimes. 
C'est pourquoi nous avons réalisé une série d'expériences dont les premières, ici rapportées, ont porté sur le rat et fournissent des résultats qui doivent nous permettre d'orienter les recherches sur le Porc. Elles avaient pour but de déterminer :

1. Si le rat, lorsqu'on lui offre ad libitum l'azote et l'énergie, est capable par luimême de consommer la quantité d'azote nécessaire et suffisante pour couvrir son besoin et de réaliser l'équilibre optimum entre l'énergie et l'azote.

2. Si le fait de modifier le niveau azoté pour une protéine donnée exerce sur la consommation spontanée d'énergie du rat des effets analogues à ceux que l'on observe chez le poulet en administrant à un niveau constant des protéines de valeur bilogique différente.

\section{I. Étude de l'ingestion spontanée d'éléments énergétiques et de protéines chez le rat en croissance par la méthode du libre choix (Expérience A).}

L'expérience décrite, qui a duré 8 semaines, a porté sur 3 lots de 9 rats $(5$ mâles et 4 femelles). Chaque animal a reçu ad libitum, dans deux mangeoires séparées, un régime protidique et un régime protéiprive. Le régime protidique contenait une source azotée variable selon les lots : farine de poisson, tourteau d'arachide, gluten de blé (tableau 1). Le régime protéiprive était composé de sucre, huile, cellulose, minéraux et vitamines.

\section{TABleaU I}

Composition des régimes.

\begin{tabular}{|c|c|c|c|c|}
\hline \multirow{2}{*}{ Sources azotées } & \multicolumn{3}{|c|}{ Régime protéique } & \multirow{2}{*}{$\begin{array}{l}\text { Régime } \\
\text { protéiprive }\end{array}$} \\
\hline & $\begin{array}{c}\text { Farine } \\
\text { de poisson }\end{array}$ & $\begin{array}{l}\text { Tourteau } \\
\text { d'arachide }\end{array}$ & $\begin{array}{l}\text { Gluten } \\
\text { de blé }\end{array}$ & \\
\hline Farine de poisson $(g) \ldots$ & 1.000 & - & 一 & - \\
\hline Tourteau d'arachide (g). & - & 1.360 & 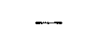 & 一 \\
\hline Gluten de blé $(g) \ldots \ldots \ldots \ldots \ldots \ldots$ & - & - & 940 & 一 \\
\hline Sucre $(g) \ldots \ldots \ldots \ldots \ldots \ldots \ldots$ & 835 & 475 & 895 & 835 \\
\hline Huile d'arachide ${ }^{1}(\mathrm{~g}) \ldots \ldots \ldots \ldots \ldots$ & 90 & 90 & 90 & 90 \\
\hline Cellulose $(g) \ldots \ldots \ldots \ldots \ldots \ldots \ldots$ & 20 & 20 & 20 & 20 \\
\hline Mélange salin (Osborne et MendeL) (g). & 45 & 45 & 45 & 45 \\
\hline Mélange vitaminique $^{2}(\mathrm{~g}) \ldots \ldots \ldots \ldots$ & 10 & 10 & 10 & 10 \\
\hline
\end{tabular}

1. Les vitamines $A$ et $D$ sont incorporées à l'huile d'arachide $(4 \mathrm{ml}$ de "vitadone $B y l a$ pour $1.000 \mathrm{ml}$ d'huile).

2. La biotine et la choline sont introduites à part sous forme de solution à raison de $5 \mathrm{ml}$ d'une solution à 20 p. 100 de choline et $5 \mathrm{ml}$ d'une solution à 0,040 p. 1.000 de biotine par $\mathrm{kg}$ d'aliment. La composition du mélange vitaminique est donné par ailleurs (HENRY et RERAT, 1963.)

Les résultats moyens généraux sont rapportés dans le tableau 2.

Le gain moyen quotidien pendant les 8 semaines d'expérience est d'autant plus élevé que la protéine est de meilleure qualité. La meilleure croissance est obtenue avec la farine de poisson, la moins bonne avec le gluten de blé, tandis que le tourteau d'ara- 
CONSOMMATION SPONTANÉE D'ÉNERGIE CHEZ LE RAT

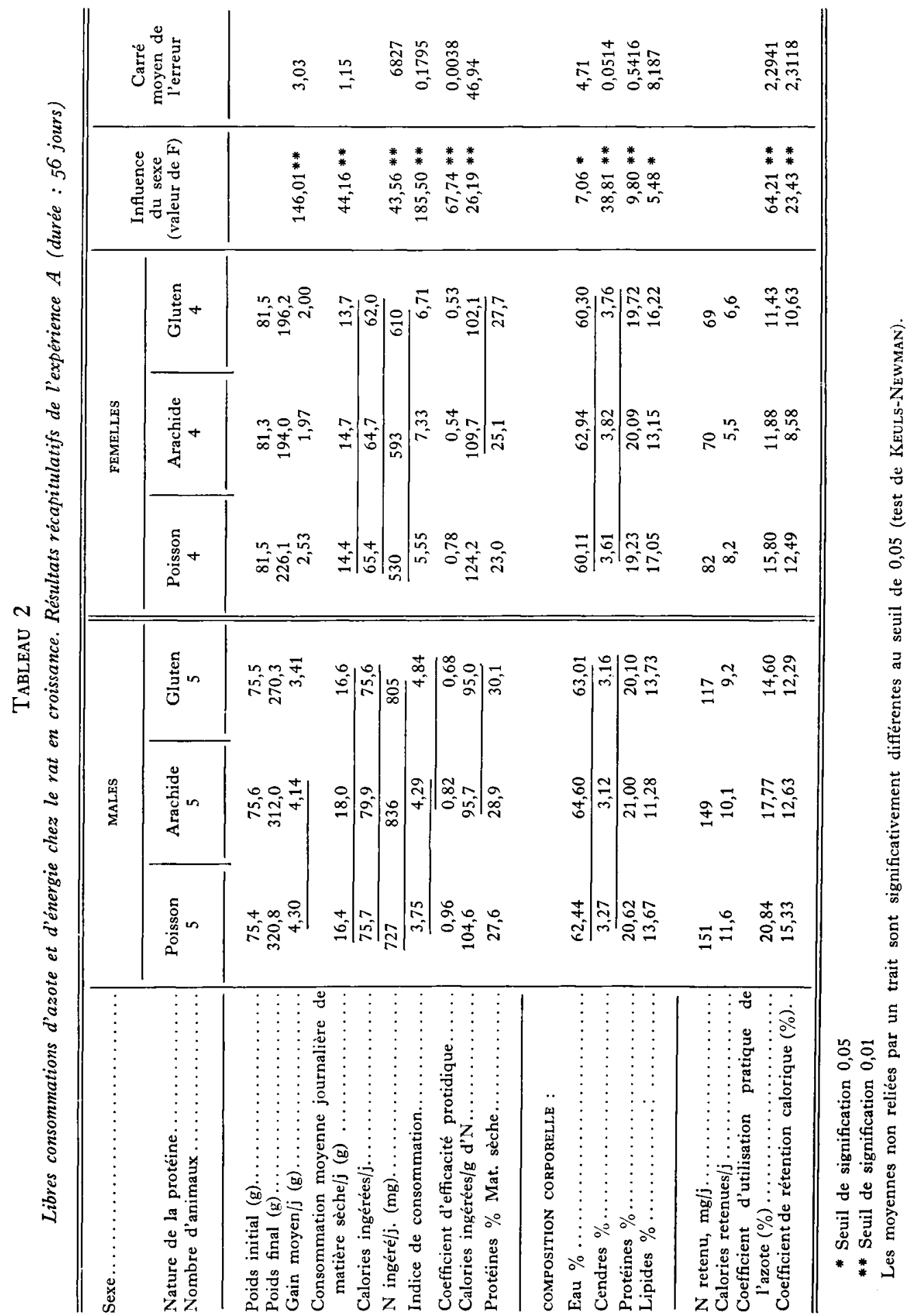


chide permet une croissance intermédiaire. Seuls les lots extrêmes sont significativement différents.

Les consommations journalières moyennes de matière sèche et d'azote ne sont pas significativement différentes lorsque varie la nature de la protéine; l'ingestion de protéines s'est équilibrée pour tous les lots à $30 \%$ environ de l'ingestion de matière sèche; cependant, on peut noter une légère augmentation de la consommation d'azote chez les animaux recevant des protéines de qualité médiocre (arachide, gluten) par rapport à ce qui se passe chez les animaux recevant de la farine de poisson. En ce qui concerne l'indice de consommation, son évolution est la même que celle du gain moyen journalier; il n'existe de différence significative qu'entre le lot recevant le gluten et le lot recevant la farine de poisson. Les valeurs obtenues pour le coefficient d'efficacité protidique classent les protéines dans l'ordre attendu; il en est de même du coefficient d'utilisation pratique de l'azote.

Deux autres faits sont à signaler (tableau 2) :

- la composition corporelle est la même pour les animaux des trois lots, malgré des différences relativement importantes dans le poids final,

- il existe pour tous les critères une influence considérable du sexe; les animaux mâles se révèlent à tous points de vue supérieurs aux animaux femelles. Mais il n'y a aucune interaction entre la nature de la source azotée et le sexe.

De l'examen de ces résultats en comparaison avec ceux d'autres expériences personnelles menées parallèlement, il ressort que le taux d'ingestion spontanée des protéines n'est pas en relation avec la couverture du besoin azoté de croissance, quelle que soit la valeur biologique des protéines dont l'animal peut disposer. Dans le cas des protéines de haute valeur biologique, comme la farine de poisson, il se produit un certain gaspillage d'azote : la preuve en est que le taux d'ingestion de ces protéines représente $28 \%$ de la matière sèche ingérée, et que le coefficient d'utilisation pratique (C. U. P.) de l'azote est de $21 \%$; or, en régime mixte, le taux de protéines de poisson pour une croissance maximum est de $16 \%$ et le C. U.P. optimum est de $40 \%$ (régime à $8 \%$ de protéines de poisson) (Rerat et al., résultats non publiés). A l'inverse, pour une protéine de mauvaise qualité comme le gluten, la quantité ingérée spontanément est insuffisante pour couvrir les besoins : l'optimum correspond à $45 \%$ (en protéines) d'un régime mixte (RERAT et al., résultats non publiés). Cependant, il faut nuancer les conclusions que l'on peut tirer de ces faits; il est possible qu'il se produise pour les régimes protidiques un phénomène d'appétence associative; par ailleurs, il est possible que le taux protidique dans les mélanges protidiques (environ $38 \%$ ) soit insuffisant pour que le rat puisse ingérer librement une plus grande proportion d'azote qu'il ne l'a fait en réalité.

Si l'ingestion d'azote est indépendante de l'activité métabolique de l'animal, la consommation d'énergie par contre pourrait être liée au poids des animaux : lorsque l'on étudie l'évolution de cette consommation en fonction du temps, on s'aperçoit qu'elle est linéaire dans le cas de l'arachide et du gluten, et curvilinéaire dans le cas de la farine de poisson; les animaux recevant du poisson ont tendance, en fin d'expérience, à limiter leur ingestion de calories, ce qui pourrait être attribué au fait qu'ils sont alors en fin de croissance et plus lourds. C'est pourquoi nous avons étudié, après transformation logarithmique des données, la régression de la consommation journalière de calories par animal et par quinzaine, sur le poids moyen pondéré pendant la 
même période de temps. Le poids moyen pondéré a été choisi comme devant traduire une évolution plus dynamique de la croissance que le poids moyen; il est donné par la formule :

$$
P=\frac{1}{2}\left[\frac{P_{1}+P_{2}}{2}+\frac{P_{2}+P_{3}}{2}\right]=\frac{P_{1}+2 P_{2}+P_{3}}{4}
$$

$P_{1}, P_{2}$ et $P_{3}$ étant respectivement les poids des animaux au début, au milieu et à la fin de la quinzaine. Une série d'équations de régression, dont chacune correspond à un lot d'animaux, a ainsi été obtenue (tableau 3) pour un même intervalle de poids (70 à $350 \mathrm{~g}$ ). Les droites représentant ces équations sont pratiquement confondues. En d'autres termes, quelle que soit la nature de la protéine, l'ingestion calorique est proportionnelle au poids élevé à une puissance fixe (voisine de 0,35 dans l'intervalle de poids donné).

En définitive, lorsqu'on présente au rat éléments azotés et éléments énergétiques à volonté, l'équilibre optimum entre ces deux types de constituants n'est pas réalisé dans le mélange spontanément ingéré par le rat. La consommation de protéines n'est pas réglée par le niveau du besoin azoté pour une croissance maximum. Par contre, la consommation d'aliments énergétiques est fonction du poids vif et s'ajuste sur la couverture du besoin énergétique.

\section{II. Étude de l'ajustement de la consommation spontanée d'aliments énergétiques en fonction de l'apport azoté (Série d'expériences $B$ ).}

La série d'expériences réalisée avait pour but d'étudier l'évolution de la libre consommation énergétique en fonction des variations de l'apport azoté, limité en quantité ou en qualité à un niveau inférieur à celui correspondant au besoin pour une croissance maximum.

Les trois sources azotées précédemment décrites ont été utilisées à trois niveaux différents $(0,6 \mathrm{~g}-1,2 \mathrm{~g}-1,8 \mathrm{~g}$ de protéines par jour, soit $96,192,288 \mathrm{mg}$ d'azote par jour). Les cinq expériences sont schématisées dans le tableau 4. Les résultats généraux de croissance et de consommation sont rapportés dans le tableau 5 .

\section{Gain de poids et consommation :}

Pour chacune des protéines étudiées, la croissance est d'autant plus rapide que le besoin azoté est mieux couvert :

- soit que l'on augmente le niveau d'administration de la protéine,

- soit que l'on apporte une protéine de valeur biologique plus élevée.

Pour une protéine donnée, la quantité de calories ingérées diminue quand est diminuée la quantité d'azote fournie (expériences I, II, III et V). Mais cette diminution spontanée de la quantité de calories ingérées est moins importante que la diminution imposée du niveau azoté : en conséquence, la quantité de calories ingérées par g d'azote ingéré augmente quand le niveau azoté diminue.

Quand, pour un niveau identique d'ingestion, la valeur biologique de la protéine ingérée est plus faible, la quantité de calories ingérées diminue en valeur absolue. En valeur relative, la quantité de calories ingérées par $\mathrm{g}$ d'azote offert diminue également, 
A. RERAT, Y. HENRY

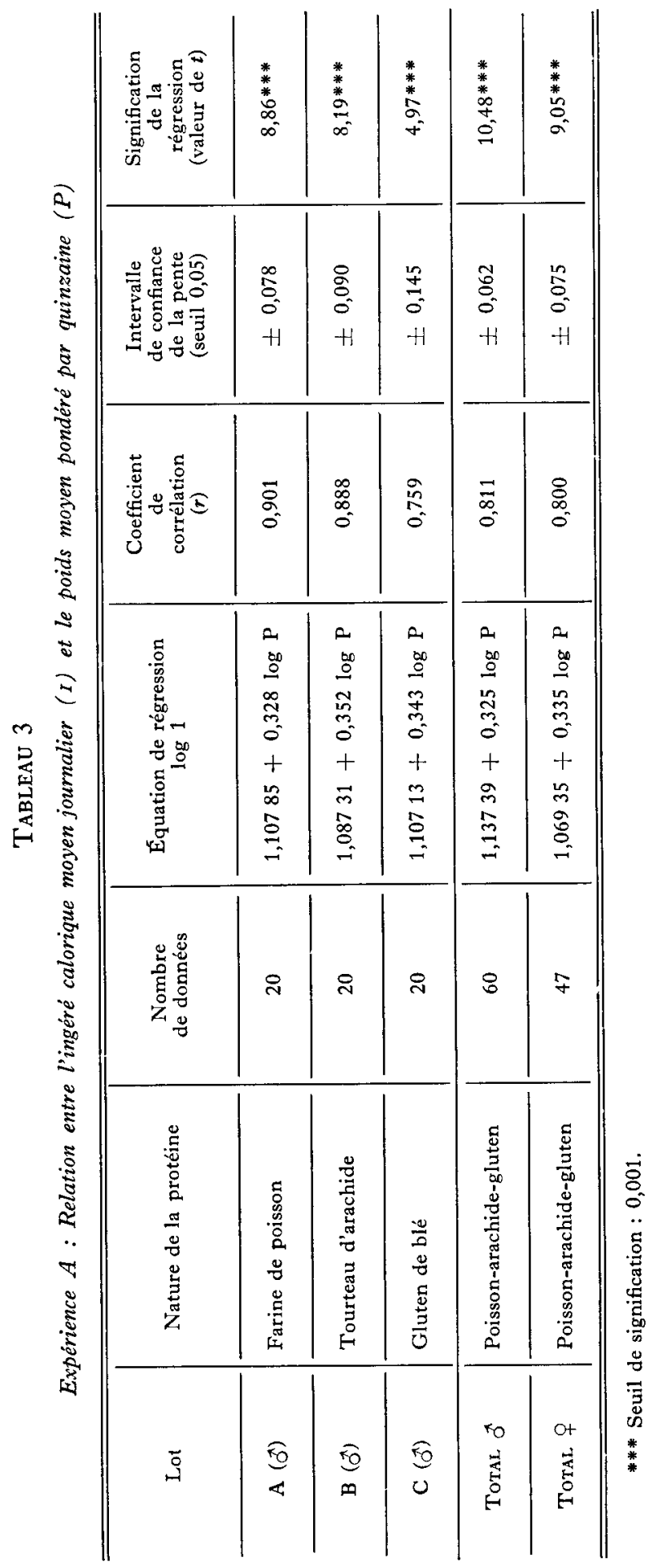


Tableau 4

Tableau descriptif des expériences (série B)

\begin{tabular}{|c|c|c|c|c|}
\hline & & Régime prot & & \\
\hline & $\begin{array}{c}\text { d'animaux } \\
\text { par lot }\end{array}$ & $\begin{array}{l}\text { Nature } \\
\text { de la protéine }\end{array}$ & $\begin{array}{c}\text { Quantité } \\
\text { de protéines/j } \\
(\mathrm{g})\end{array}$ & protéiprive \\
\hline $\begin{array}{l}\text { Expérience I } \\
\text { Farine de poisson à niveau variable. }\end{array}$ & 8 & 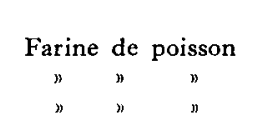 & $\begin{array}{l}1,8 \\
1,2 \\
0,6\end{array}$ & ad libitum \\
\hline $\begin{array}{l}\qquad \text { Expérience } I I \\
\text { Tourteau d'arachide à niveau va- } \\
\text { riable..................... }\end{array}$ & 10 & $\begin{array}{c}\text { Farine de poisson } \\
\text { Tourteau d'arachide } \\
\qquad n \\
n\end{array}$ & $\begin{array}{l}1,2 \\
1,8 \\
1,2 \\
0,6\end{array}$ & ad libitum \\
\hline $\begin{array}{l}\text { Expérience III } \\
\text { Gluten de blé à niveau variable.. }\end{array}$ & 9 & $\begin{array}{c}\text { Farine de poisson } \\
\text { Gluten de blé } \\
n \\
"\end{array}$ & $\begin{array}{l}1,2 \\
1,8 \\
1,2 \\
0,6\end{array}$ & ad libitum \\
\hline 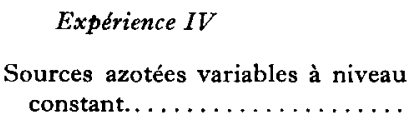 & 12 & $\begin{array}{l}\text { Farine de poisson } \\
\text { Tourteau d'arachide } \\
\text { Gluten de blé }\end{array}$ & $\begin{array}{l}1,2 \\
1,2 \\
1,2\end{array}$ & ad libitum \\
\hline $\begin{array}{l}\text { Farine de poisson et tourteau } \\
\text { d'arachide à } 3 \text { niveaux......... }\end{array}$ & 8 & $\begin{array}{c}\text { Farine de poisson } \\
" \\
\text { Tourteau d'arachide } \\
", \\
"\end{array}$ & $\begin{array}{l}1.8 \\
1,2 \\
0,6 \\
1,8 \\
1,2 \\
0,6\end{array}$ & ad libitum \\
\hline
\end{tabular}

à l'inverse de ce qui se passe lorsque varie la quantité d'azote ingérée. La contradiction entre ces deux phénomènes n'est qu'apparente : elle s'explique par le fait que, quand on fait varier la qualité des protéines, le pourcentage d'azote retenu par g d'azote ingéré est variable, alors que, quand on fait varier la quantité d'une protéine donnée, il est relativement constant. Il en résulte que, si l'on rapporte l'ingéré calorique à l'azote retenu, il augmente dans tous les cas où est diminuée la quantité d'azote utilisable, que ce soit par l'intermédiaire de la quantité de protéine administrée, ou à quantité égale, en utilisant une protéine de plus faible valeur biologique.

En ce qui concerne le coefficient d'efficacité protidique ainsi que le coefficient d'utilisation pratique de l'azote, on retrouve, en régime séparé, des variations de sens ana- 


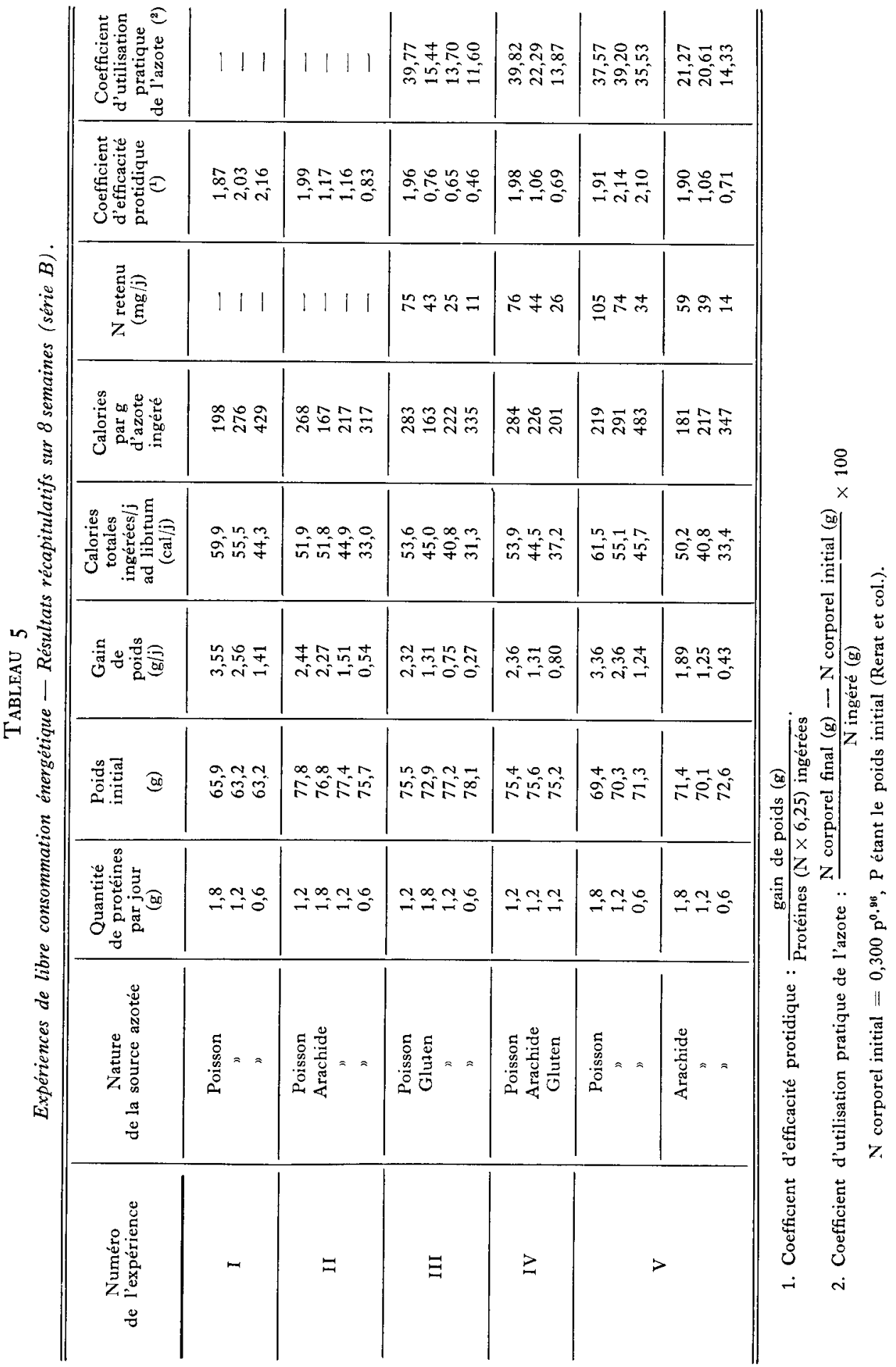


logue à celles qui sont enregistrées en régime mixte quand on modifie l'administration d'azote en qualité et en quantité. La hiérarchie des protéines est celle trouvée classiquement : poisson, arachide, gluten. Cependant, ces variations, pour une protéine donnée à différents niveaux, sont beaucoup plus faibles que celles constatées en régime mixte à différents taux (RERAT et HENRY, résultats non publiés).

Enfin, il faut noter, d'une expérience à l'autre, la grande constance des résultats pour la même quantité d'une même protéine. Ceci est d'autant plus remarquable que les cinq expériences se sont déroulées sur une durée de plus d'un an.

\section{Composition corporelle :}

Comme le montre le tableau 6, les résultats de composition corporelle ne sont pas très différents d'un lot à l'autre, de même que d'une expérience à l'autre, malgré des variations importantes dans le poids final. Si l'on rapproche ces résultats de ceux obtenus en régime complet, avec les mêmes sources azotées et à des niveaux comparables d'ingestion, on constate que les animaux en libre consommation énergétique ont tendance à être plus maigres que ceux en régime imposé (RERAt et HenRY, résultats non publiés). Cependant, les expériences n'ayant pas été effectuées en même temps, cette hypothèse nécessite une vérification.

\section{Relation entre la consommation spontanée d'énergie et le poids :}

L'analyse détaillée du tableau 5 montre que, lorsque les gains de poids sont les mêmes, en général la consommation d'énergie est la même quelle que soit l'origine de 1 a protéine et son niveau : c'est le cas des lots " poisson : 0,6 ", " arachide : 1,2 " et " glu-

\section{Tablfau 6}

Expériences de libre consommation énergétique - Résultats de composition corporelle après 8 semaines (Série $B$ ).

\begin{tabular}{|c|c|c|c|c|c|c|c|}
\hline $\begin{array}{l}\text { Numéro de } \\
\text { l'expérience }\end{array}$ & $\begin{array}{l}\text { Nature de la } \\
\text { source azotée }\end{array}$ & $\begin{array}{l}\text { Quantité de } \\
\text { protéines/j. }\end{array}$ & $\begin{array}{l}\text { Poids final } \\
\text { (g) }\end{array}$ & $\begin{array}{c}\text { Eau } \\
\%\end{array}$ & $\begin{array}{c}\text { Cendres } \\
\%\end{array}$ & $\begin{array}{c}\text { Protéines } \\
\%\end{array}$ & $\begin{array}{c}\text { Lipides } \\
\%\end{array}$ \\
\hline III & $\begin{array}{c}\text { Poisson } \\
\text { Gluten } \\
" \\
"\end{array}$ & $\begin{array}{l}1,2 \\
1,8 \\
1,2 \\
0,6\end{array}$ & $\begin{array}{r}207,8 \\
147,5 \\
120,2 \\
93,6\end{array}$ & $\begin{array}{l}63,05 \\
65,81 \\
64,65 \\
64,70\end{array}$ & $\begin{array}{l}3,39 \\
3,78 \\
4,00 \\
4,44\end{array}$ & $\begin{array}{l}19,39 \\
19,09 \\
18,85 \\
18,78\end{array}$ & $\begin{array}{l}14,17 \\
11,31 \\
12,49 \\
12,33\end{array}$ \\
\hline IV & $\begin{array}{l}\text { Poisson } \\
\text { Arachide } \\
\text { Gluten }\end{array}$ & $\begin{array}{l}1,2 \\
1,2 \\
1,2\end{array}$ & $\begin{array}{l}209,8 \\
150,1 \\
120,6\end{array}$ & $\begin{array}{l}63,98 \\
65,52 \\
64,93\end{array}$ & $\begin{array}{l}3,54 \\
3,75 \\
4,14\end{array}$ & $\begin{array}{l}19,02 \\
19,04 \\
18,22\end{array}$ & $\begin{array}{l}13,46 \\
11,69 \\
12,71\end{array}$ \\
\hline \multirow{2}{*}{$\mathrm{V}$} & $\begin{array}{c}\text { Poisson } \\
n \\
n\end{array}$ & $\begin{array}{l}1,8 \\
1,2 \\
0,6\end{array}$ & $\begin{array}{l}257,5 \\
211,9 \\
140,9\end{array}$ & $\begin{array}{l}62,67 \\
61,49 \\
61,19\end{array}$ & $\begin{array}{l}3,36 \\
3,49 \\
3,81\end{array}$ & $\begin{array}{l}19,20 \\
18,20 \\
17,39\end{array}$ & $\begin{array}{l}14,77 \\
16,82 \\
17,61\end{array}$ \\
\hline & $\begin{array}{c}\text { Arachide } \\
" \\
"\end{array}$ & $\begin{array}{l}1,8 \\
1,2 \\
0,6\end{array}$ & $\begin{array}{r}177,3 \\
140,4 \\
96,7\end{array}$ & $\begin{array}{l}64,23 \\
63,54 \\
64,50\end{array}$ & $\begin{array}{l}3,51 \\
3,78 \\
4,41\end{array}$ & $\begin{array}{l}18,86 \\
18,58 \\
18,38\end{array}$ & $\begin{array}{l}13,40 \\
14,10 \\
12,71\end{array}$ \\
\hline
\end{tabular}


ten : 1,8 "; il en est de même des lots " poisson : 1,2 » et " arachide : 1,8 ». On peut donc supposer qu'il existe une relation étroite entre l'ingéré calorique et le niveau de croissance. C'est pourquoi, comme dans l'expérience précédemment décrite, nous avons recherché l'existence d'une relation logarithmique entre ingéré calorique et poids vif pondéré, chacune de ces données étant calculée pour des durées de deux semaines. Une série d'équations a ainsi été obtenue dont chacune correspond aux valeurs obtenues dans un des lots (tableau 7). Les droites représentatives de ces équations (fig. 1 et 2) sont pratiquement confondues; par conséquent, quels que soient la nature de la protéine et son niveau d'ingestion, la consommation spontanée d'énergie est proportionnelle au poids élevé à une puissance fixe (voisine de 0,5 dans l'intervalle de poids $60-150 \mathrm{~g}$ ). Bien qu'il ne soit pas possible de distinguer statistiquement les équations correspondant aux différentes protéines, il faut préciser que la droite correspondant à la meilleure protéine (farine de poisson) se trouve toujours au dessus de la droite correspondant à l'arachide ou au gluten; il est possible qu'il existe là un phénomène dont la signification nous échappe. Deux hypothèses peuvent être faites : pour calculer les calories brutes ingérées, il a fallu utiliser certains coefficients dont la portée n'est peut-être pas universelle; par ailleurs, le mode de représentation du poids vif ne tient peut-être pas assez compte de la vitesse de croissance : dans ces conditions, les animaux au poisson, ayant eu une croissance plus rapide, ingéreraient plus de calories pour un poids donné.

La valeur de l'exposant obtenu est supérieure à celle obtenue dans la première expérience décrite $(0,32$ à 0,35$)$; mais dans ce cas, l'exposant était calculé pour un poids oscillant entre 70 et $350 \mathrm{~g}$, très différent des poids pour lesquels ont été faits les calculs dans la première série.

En fin de compte, on peut conclure, comme dans l'expérience précédente, que, chez le rat, la consommation d'énergie est ajustée en fonction du poids et du gain de poids permis par les protéines.

\section{Relation entre la consommation spontanée d'énergie et la rétention azotée :}

Étant donné que le gain de poids est lui-même fonction de la quantité et de la qualité des protéines ingérées, c'est-à-dire de la quantité d'azote fixée, l'évolution de la consommation spontanée d'énergie a été étudiée directement en fonction de la quantité d'azote retenue dans la carcasse, pour chacune des protéines aux 3 niveaux et pour les 3 protéines à niveau constant. Une série de droites de régression a ainsi été obtenue (fig. 3), dont les équations sont données dans le tableau 8. On remarque que ces droites de régression sont pratiquement confondues, tant par leurs ordonnées à l'origine que par leurs pentes; la régression linéaire est dans tous les cas hautement significative. Autrement dit, quelles que soient la quantité et la nature de la source azotée, la consommation spontanée d'énergie est directement proportionnelle à la quantité d'azote fixée par l'animal pour fabriquer ses tissus de croissance; en d'autres termes, le rat ingère une quantité constante de calories par g d'azote supplémentaire retenu (environ 300 calories). Cette conclusion n'est valable que dans nos conditions expérimentales, c'est-à-dire pour des animaux dont le besoin d'entretien a été pratiquement comparable d'une expérience à l'autre (durée expérimentale identique, poids moyen des lots peu différent) et dont le besoin azoté pour une croissance optimum n'est pas couvert. On ne saurait l'extrapoler à des animaux placés dans des conditions expérimentales très diffé- 


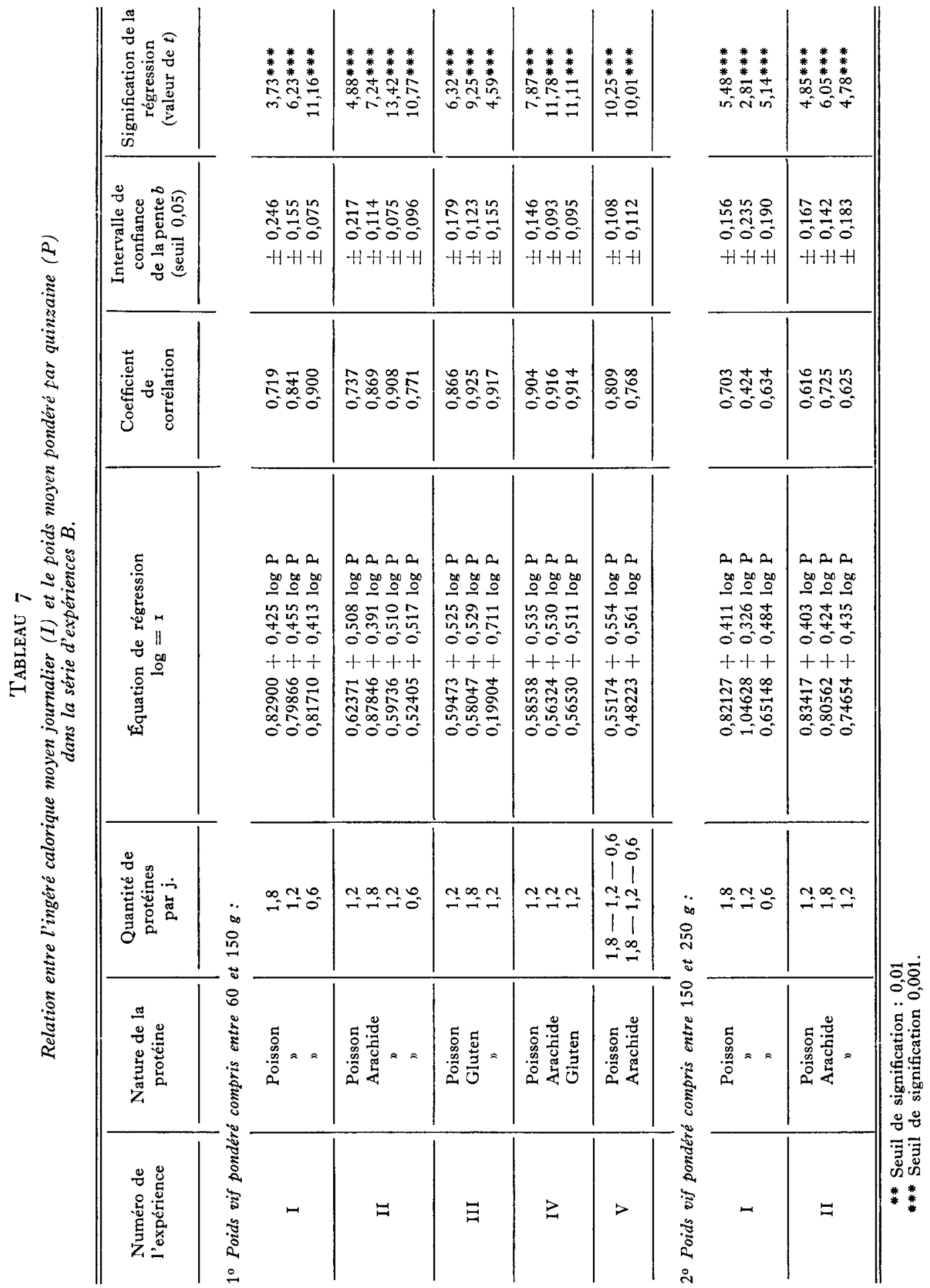




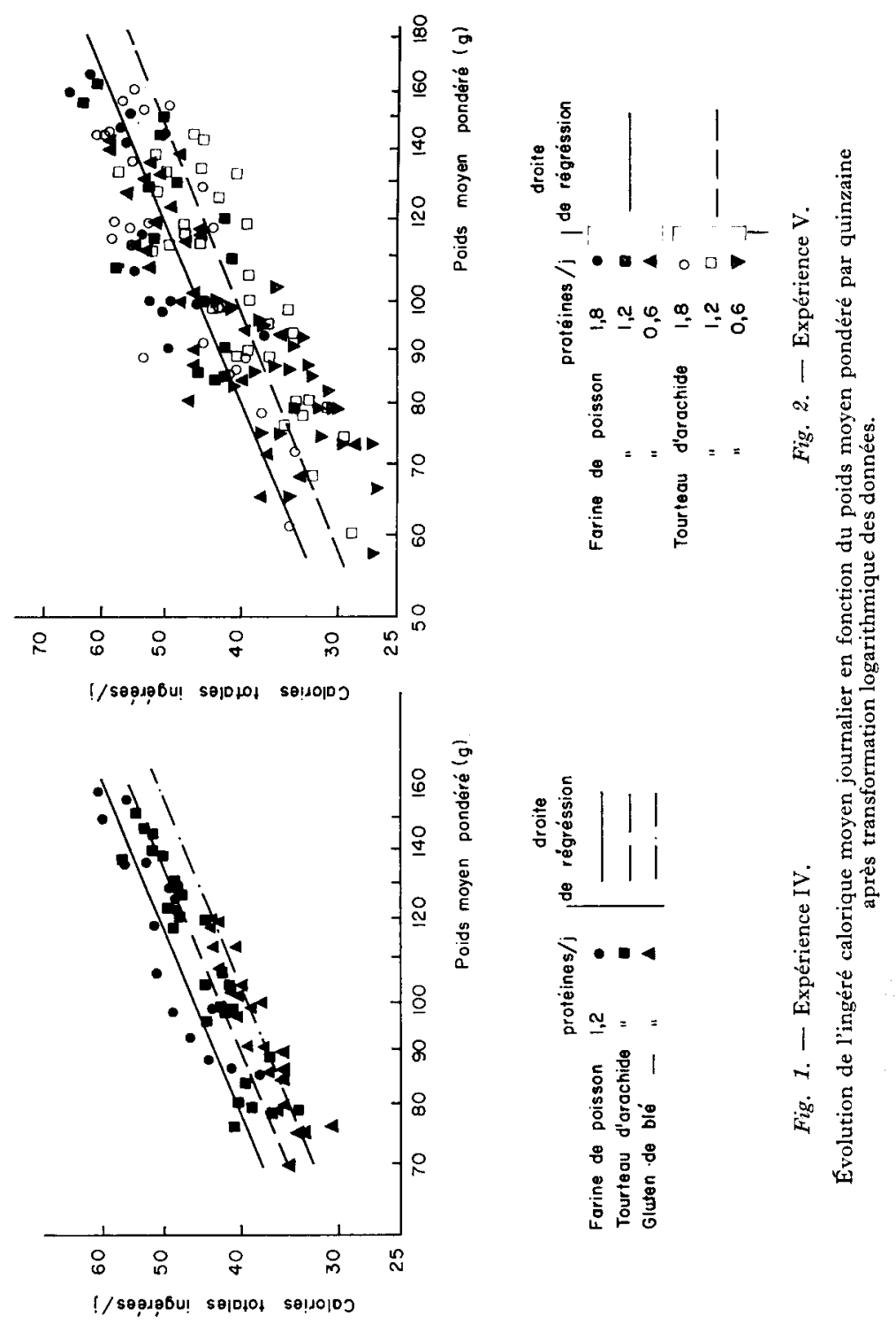




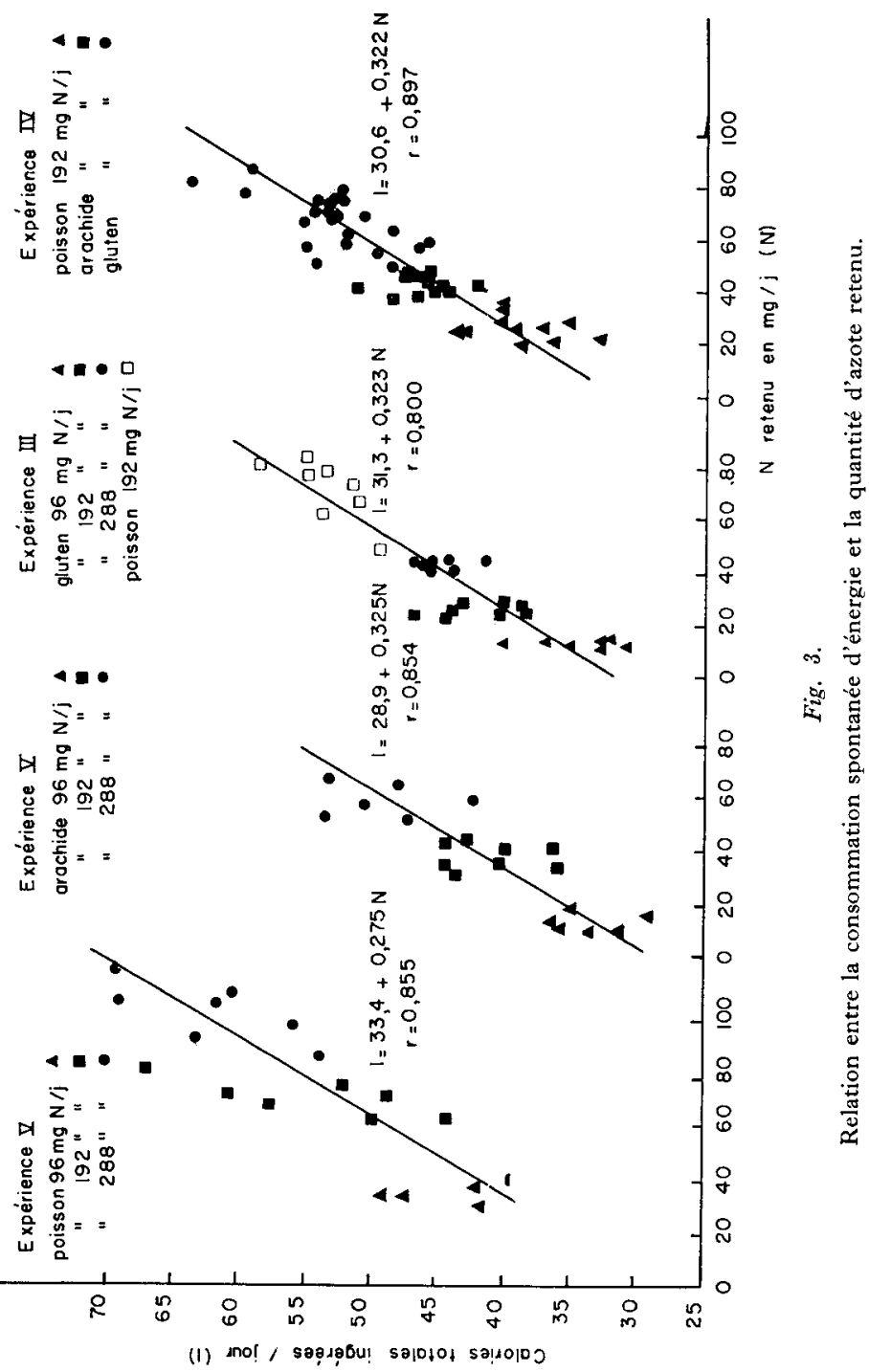


A. RÉRAT, Y. HENRY

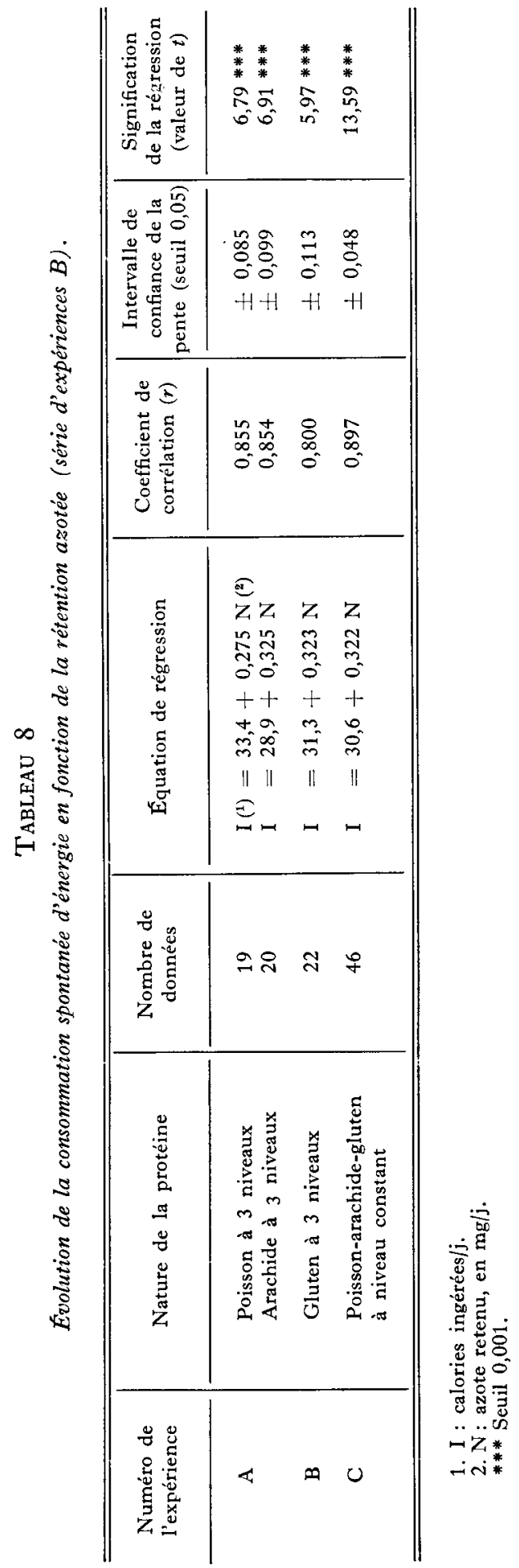


rentes. La preuve en est que, pour les animaux qui reçoivent azote et énergie ad libitum (ensemble des animaux de la première expérience décrite : expérience $\mathrm{A}$ ), la relation entre azote retenu et calories ingérées est très différente et traduite par l'équation :

$$
\mathrm{I}=49,8+0,187 \mathrm{~N} \text {, }
$$

le coefficient de corrélation étant élevé $(r=0,94)$; il faut noter que la rétention brute journalière d'azote dans ces carcasses a oscillé entre $70 \mathrm{mg}$ et $150 \mathrm{mg}$, alors que pour les animaux ayant reçu un apport d'azote restreint, cette rétention variait entre 11 et $105 \mathrm{mg}$. Dans le premier cas, le poids moyen et, par voie de conséquence, le besoin d'entretien des animaux était beaucoup plus élevé; on conçoit que, comme cela est le cas pour la relation ingéré calorique-poids vif, la pente de la droite traduisant les relations entre ingéré calorique et azote retenu soit plus faible pour les animaux les plus lourds.

Quoi qu'il en soit, la relation constante, indépendante de la valeur biologique des protéines, qui existe entre l'ingéré calorique et la rétention azotée (rétentions moyennes journalières comprises entre 10 et $100 \mathrm{mg}$ ) permet d'établir que c'est la fraction des protéines utilisable à des fins de croissance qui détermine le niveau de consommation énergétique.

\section{CONCLUSIONS}

En définitive, il est possible de conclure que la consommation de nourriture est réglée, chez le rat en croissance, par le besoin énergétique, lequel est fonction du niveau de croissance et du degré de rétention azotée permis par la qualité et la quantité des protéines ingérées par l'animal.

Par contre, il ne semble exister aucune relation entre le niveau du besoin azoté et la consommation spontanée de protéines. Si le rapport calories/protéines calculé dans le régime ingéré spontanément par l'animal ne semble être soumis à aucune règle précise lorsque l'apport azoté est fourni à volonté, il n'en est pas de même quand l'apport azoté est réduit : ce rapport augmente quand diminue l'apport azoté, et diminue quand s'abaisse la valeur biologique des protéines.

\section{RÉFÉRENCES BIBLIOGRAPHIQUES}

Abraham J., Calet C., Rerat A., Jacquot R., 1961. Solidarité des besoins énergétique et protéique de croissance : l'ajustement spontané des calories et des protéines. C. R. Acad. Sci. ,Paris, 253, 2768-2770.

Calet C., Jouandet C., Baratou J., 1961. Variation de la consommation spontanée d'énergie du poussin en fonction de la nature des matières azotées du régime. Ann. Biol. anim. Bioch. Biophys., 1, 1-2.

HenRy Y., Rerat A., 1962. Influence de la qualité et de la quantité des matières azotées ingérées sur la consommation spontanée d'énergie chez le rat en croissance. Ann. Biol. anim. Bioch. Biophys., 2, 267-276.

Henry Y., Rerat A., 1963. Étude de l'ingestion spontanée d'éléments énergétiques et de protéines chez le le rat en croissance par la méthode du libre choix. Ann. Biol. anim. Bioch. Biophys. 3, 103-117.

Rerat A., Henry Y., JACQuot R., 1962. Relation entre la consommation spontanée d'énergie et la rétention azotée chez le rat en croissance. C. R. Acad. Sci., Paris, 256, 787-789. 